\title{
Loss of CDX2 in colorectal cancer is associated with histopathologic subtypes and microsatellite instability but is prognostically inferior to hematoxylin-eosin-based morphologic parameters from the WHO classification
}

Björn Konukiewitz ${ }^{1,2}$, Maxime Schmitt ${ }^{1}$, Miguel Silva ${ }^{3}$, Junika Pohl ${ }^{1}$, Corinna Lang ${ }^{1}$, Katja Steiger (D ${ }^{1}$, Kathrin Halfter ${ }^{4}$, Jutta Engel $^{4}$, Anna Melissa Schlitter ${ }^{1}$, Melanie Boxberg ${ }^{1}$, Nicole Pfarr ${ }^{1}{ }^{1}$, Dirk Wilhelm ${ }^{5}$, Sebastian Foersch ${ }^{6}$, Markus Tschurtschenthaler (iD ${ }^{3,7}$, Wilko Weichert ${ }^{1,8,9}$ and Moritz Jesinghaus iD $^{1,10^{凶}}$

(c) The Author(s) 2021

BACKGROUND: Immunohistochemical loss of CDX2 has been proposed as a biomarker of dismal survival in colorectal carcinoma (CRC), especially in UICC Stage II/III. However, it remains unclear, how CDX2 expression is related to central hematoxylin-eosin (HE)based morphologic parameters defined by 2019 WHO classification and how its prognostic relevance is compared to these parameters.

METHODS: We evaluated CDX2 expression in 1003 CRCs and explored its prognostic relevance compared to CRC subtypes, tumour budding and WHO grade in the overall cohort and in specific subgroups.

RESULTS: CDX2-low/absent CRCS were enriched in specific morphologic subtypes, right-sided and microsatellite-instable (MSI-H) CRCs $(P<0.001)$ and showed worse survival characteristics in the overall cohort/UICC Stage II/III (e.g. DFS: $P=0.005)$ and in microsatellite stable and left-sided CRCs, but not in MSI-H or right-sided CRCs. Compared with CDX2, all HE-based markers showed a significantly better prognostic discrimination in all scenarios. In multivariate analyses including all morphologic parameters, CDX2 was not an independent prognostic factor.

CONCLUSION: CDX2 loss has some prognostic impact in univariate analyses, but its prognostic relevance is considerably lower compared to central HE-based morphologic parameters defined by the WHO classification and vanishes in multivariate analyses incorporating these factors.

British Journal of Cancer (2021) 125:1632-1646; https://doi.org/10.1038/s41416-021-01553-0

\section{INTRODUCTION}

Colorectal carcinoma (CRC) is a major cause of cancer-related deaths worldwide and is currently the third most common cancer among women and men in the United States [1, 2]. Histologically, $\mathrm{CRC}$ is a heterogeneous tumour entity. Besides the conventional adenocarcinoma NOS, a variety of histopathological CRC subtypes are known [3-12], which are usually given in routine pathology reports alongside other well-established morphological parameters, such as tumour budding or WHO grade. CRC subtypes, tumour budding and WHO grade are (mainly) assessed through the evaluation of hematoxylin-eosin (HE)-stained slides and are listed as essential diagnostic parameters for CRC in the WHO classification of tumours of the digestive system manual (WHO classification) [13]. Recently, we demonstrated the high prognostic relevance of these morphologic parameters provided by the recent WHO classification (CRC subtypes, tumour budding, WHO grade) in a large cohort of $>1000$ CRCs [8].

Caudal-related homeobox transcription factor 2 (CDX2) is a highly conserved master transcription factor that controls the development and differentiation of the intestinal epithelium $[14,15]$. CDX2 is normally assessed via immunohistochemistry (IHC) with CDX2 being diffusely expressed in non-neoplastic colorectal mucosa and in the majority of CRCs [16]. Loss of CDX2 expression has been proposed as a biomarker of dismal clinical

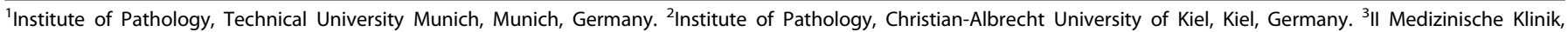

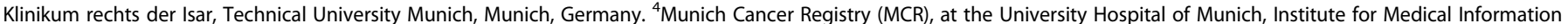

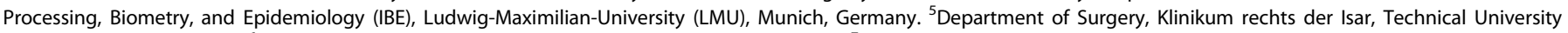

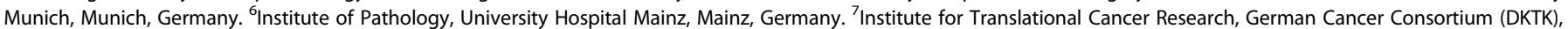

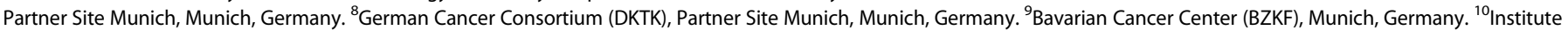
of Pathology, University Hospital Marburg, Marburg, Germany. ${ }^{凶}$ email: moritz.jesinghaus@uni-marburg.de 
outcomes in CRC. Dalerba et al. reported that CDX2 loss might not only identify CRC patients with poorer survival characteristics but also those with an especially aggressive disease course in specific stage groups (e.g. UICC Stage II/III) [17]. While the general impact on survival of CDX2 loss has been confirmed by additional studies [18-26], it remains to be seen how CDX2 expression is related to the aforementioned HE-based morphologic factors and how the prognostic relevance of $\mathrm{CDX} 2$ ranks compared to these central morphologic parameters.

In order to address these questions, we evaluated CDX2 expression in a large cohort of over 1000 CRCs and analysed its connection to tumour budding, WHO grade and the various histomorphological CRC subtypes and compared the impact of all factors in the overall cohort, specific stage groups, right- vs. leftsided tumours as well as in microsatellite subgroups and finally in multivariate analyses incorporating all factors.

\section{PATIENTS AND METHODS \\ Cohort}

The investigated cohort comprised 1003 CRC patients that underwent surgical resection between 1997 and 2018 at the University Hospital Klinikum Rechts der Isar of the Technical University of Munich, Germany. Patients signed a general informed consent protocol during admission to the hospital. All patients with colorectal carcinomas from this time span with fully available clinicopathological/survival data as well as full block tumour tissue and from which tumour tissue was present on the constructed tissue microarray were included in this study. Tumours with other neoplasms of the colorectal system (e.g. neuroendocrine tumours, non-epithelial tumours etc.) were excluded. One case of an undifferentiated carcinoma from the original cohort was also excluded to avoid statistical bias. The clinicopathological characteristics, as well as survival data for all patients, were extracted from hospital records and from the Munich Cancer Registry. For overall survival (OS), all recorded patient deaths were noted, while for disease-specific survival (DSS) only tumour-associated deaths were recorded as events. Loco-regional or distant recurrence was noted as an event for disease-free survival (DFS). Endpoints of all survival comparisons were either events or a loss of follow-up, in which case the patients were censored at the time of the last available entry regarding the specific patient either in hospital records or in the Munich Cancer Registry. The treatment concepts of included patients followed internal policies, which were based on the given German guidelines at the time of diagnosis, generally meaning that all patients were intended to receive stage-adapted treatment. The definitive therapy regimen for each individual was decided by a multidisciplinary team of physicians during a specialised gastrointestinal tumour board. In most cases of colon cancer, this meant primary resection in all cases and adjuvant therapy in UICC Stage III. For Stage UICC II, adjuvant chemotherapy was only administered in high-risk patients (pT3/ $4, G 3,<70$ years). For rectal cancers, neoadjuvant RCTx served as the standard for advanced cases (UT3N+) of the middle or lower third of the rectum, while non-advanced cancers and tumours of the proximal third of the rectum generally received primary surgery, followed by adjuvant chemotherapy depending on the post-operative tumour stage. The local ethics committee of the Technical University of Munich approved this study (reference number: 252/16s).

\section{Evaluation of CDX2 expression}

CDX2 expression was evaluated in 1003 CRCs via CDX2 immunohistochemistry using a tissue microarray harbouring two tumour-carrying cores from each carcinoma. An automated immunostainer (BOND RXm System, Leica Biosystems, Germany) was used for immunohistochemical staining of CDX2 (clone: EPR2764Y, dilution: 1:500, Abcam, UK) on 2- $\mu \mathrm{m}$ sections from a tissue microarray. Briefly, after deparaffinization, antigen retrieval was performed with Epitope Retrieval 1 (Leica Biosystems, Germany; equivalent to citrate buffer $\mathrm{pH}$ ) for $20 \mathrm{~min}$ and antibody binding was detected using a Polymer Refine Detection Kit (Leica Biosystems, Germany) without postprimary antibody and hematoxylin counterstain. Appropriate positive and negative controls were run in parallel. The evaluation of CDX2 expression was performed manually by two experienced Gl pathologists (MJ, BK) blinded to clinicopathological parameters.

Nuclear staining of CDX2 was considered specific. For each tumour, the number of positive tumour cells was counted and a cumulative percentage score for both cores was assigned for each CRC (range: 0-100\%), for each tumour a minimum of 500 tumour cells were counted. The pattern of CDX2 expression was also analysed and divided into three classes: absent if no staining was visible, heterogeneous if areas with a complete loss of staining were noted and diffuse, if the tumours either showed a complete expression in $100 \%$ of the tumour cells or only a CDX2 loss in intermingled cells. Using normal colorectal mucosa as a reference, a staining intensity comparable to normal mucosa was considered as strong, a slightly weaker but still clearly visible staining was classified as medium and a faint, barely visible staining intensity was considered as weak. When no expression was detectable, the staining intensity was classified as absent.

\section{Evaluation of HE-based morphologic parameters}

After we screened the CRCs included in our cohort for their expression of CDX2, we aimed to correlate their CDX2 expression status with $\mathrm{H} \& \mathrm{E}$-based histopathological parameters. All of the tumours that were investigated for CDX2 expression in this study $(n=1003)$, received an in-depth histopathological characterisation in a previous study from our group, where this cohort was analysed by two expert Gl pathologists (MJ, WW) regarding the distribution and the prevalence of the essential morphologic criteria given in the 2019 WHO classification of colorectal carcinoma [8]. As described previously in detail, full block H\&E slides from CRC resection specimens were re-classified according to the current CRC subtypes listed in the 2019 WHO classification (adenocarcinoma NOS, mucinous adenocarcinoma, signet-ring carcinoma, medullary carcinoma, serrated adenocarcinoma, micropapillary adenocarcinoma, adenoma-like adenocarcinoma, adenosquamous carcinoma, carcinoma with sarcomatoid components, undifferentiated carcinoma, MANEC/NEC). Furthermore, all CRCs were analysed regarding their WHO grade (low grade, formerly G1/ G2 vs. high grade, formerly G3) [13] and their tumour budding activity (Bd1: low tumour budding $0-4$ buds in $\times 20$ magnification, $B d 2$ : intermediate tumour budding 5-9 buds in $\times 20$ magnification, Bd3: high tumour budding $>10$ buds in $\times 20$ magnification) [27]. Microsatellite status was available for all CRCs (assessed via IHC/PCR in our previous study) as described previously [8] (cohort details; Table 1).

\section{Statistics}

Statistical analyses were performed using SPSS version 26 (SPSS Institute, Chicago, IL) using $X^{2}$ test as well as $X^{2}$ test for trends and Fisher's exact test. The Bonferroni method was used to correct for multiple testing. Survival comparisons were performed using the Kaplan-Meier method and a logrank test was used to test the significance of survival differences. Multivariate analyses were done with the Cox proportional hazard model. All statistical tests were performed two-sided, $P$ values $\leq 0.05$ were considered significant.

\section{RESULTS}

\section{Clinicopathological characteristics}

Fifty-seven percent $(n=575)$ of the 1003 patients were male, the median patient age was 69 years. Right-sided (caecum until splenic flexure; $n=485 ; 48 \%$ ) and left-sided CRCs (descending colon until rectum; $n=518 ; 52 \%$ ) were almost evenly present. Using the eighth edition of the TNM classification of malignant tumours [28], pTNM staging was determined (207 Stage I (21\%), 326 (32\%) Stage II, 318 (32\%) Stage III and 152 (15\%) Stage IV tumours). Three hundred and twenty-two patients relapsed (32\%), 408 patients $(40.7 \%)$ died during follow-up, for 293 patients a tumour specific death was noted (cohort details: Table 1).

\section{Distribution of CDX2 expression and CDX2 groups}

Most CRCs showed a diffuse CDX2 expression (median: 100\% positive tumour cells, mean: $91 \%$ positive tumour cells) resulting in 783 CRCs (78\%) showing a diffuse nuclear expression in all tumours cells and a total of $85 \%(852 / 1003)$ showing a diffuse nuclear staining in $\geq 90 \%$ of tumour cells. Forty-seven (5\%) CRCs showed a nuclear expression in $61-89 \%$ of tumour cells and 90 carcinomas $(9 \%)$ showed a reduced CDX2 staining within the range of $1-60 \%$ positive tumour cells. A complete loss of CDX2 expression was noted for 12 CRCs (1\%). In order to perform deeper statistical analyses, we needed to find an ideal cut-off to form 
Table 1. Distribution and prognostic impact of CDX2 expression, staging parameters as well as CRC subtypes, tumour budding and WHO grade in the overall cohort.

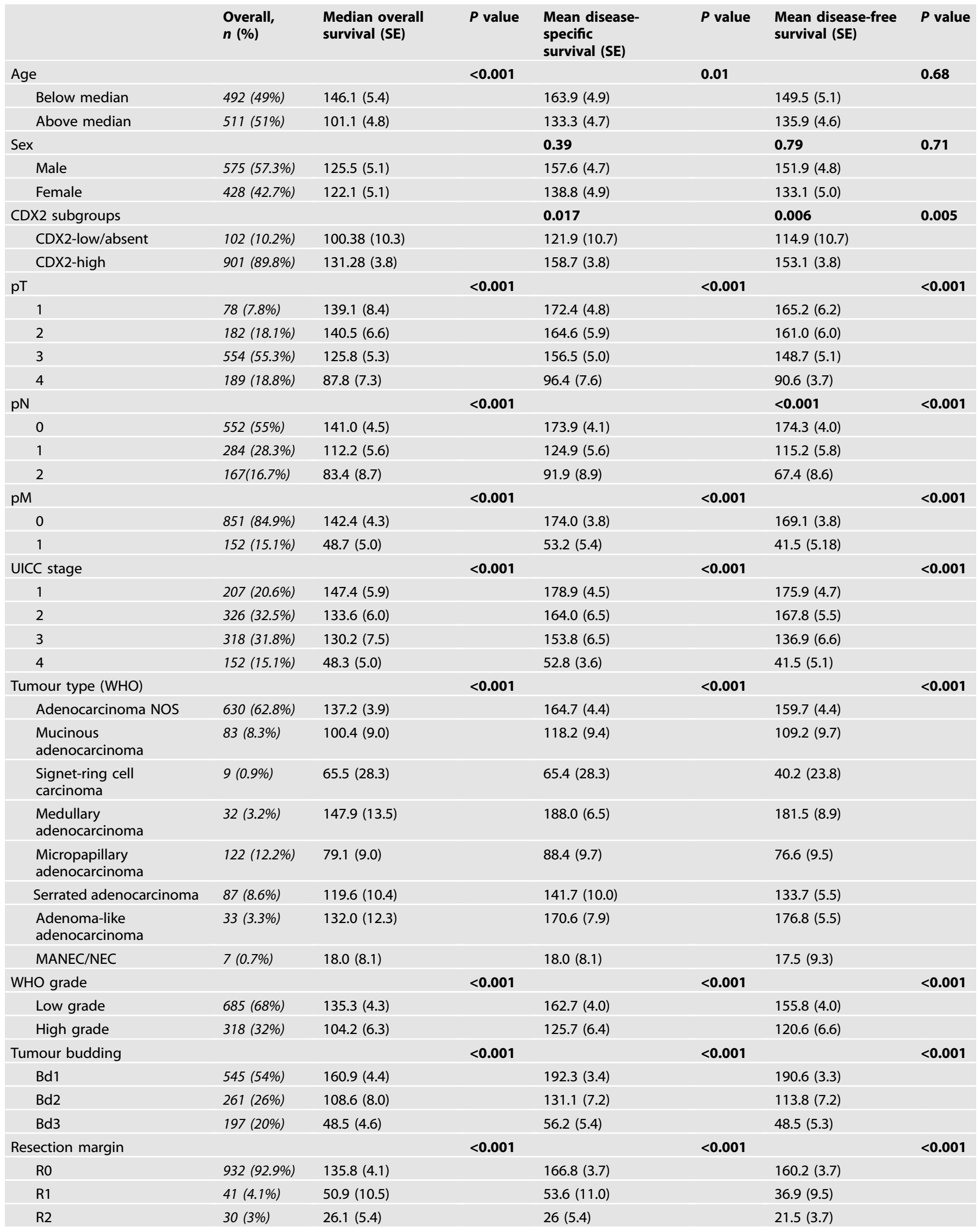


Table 1 continued

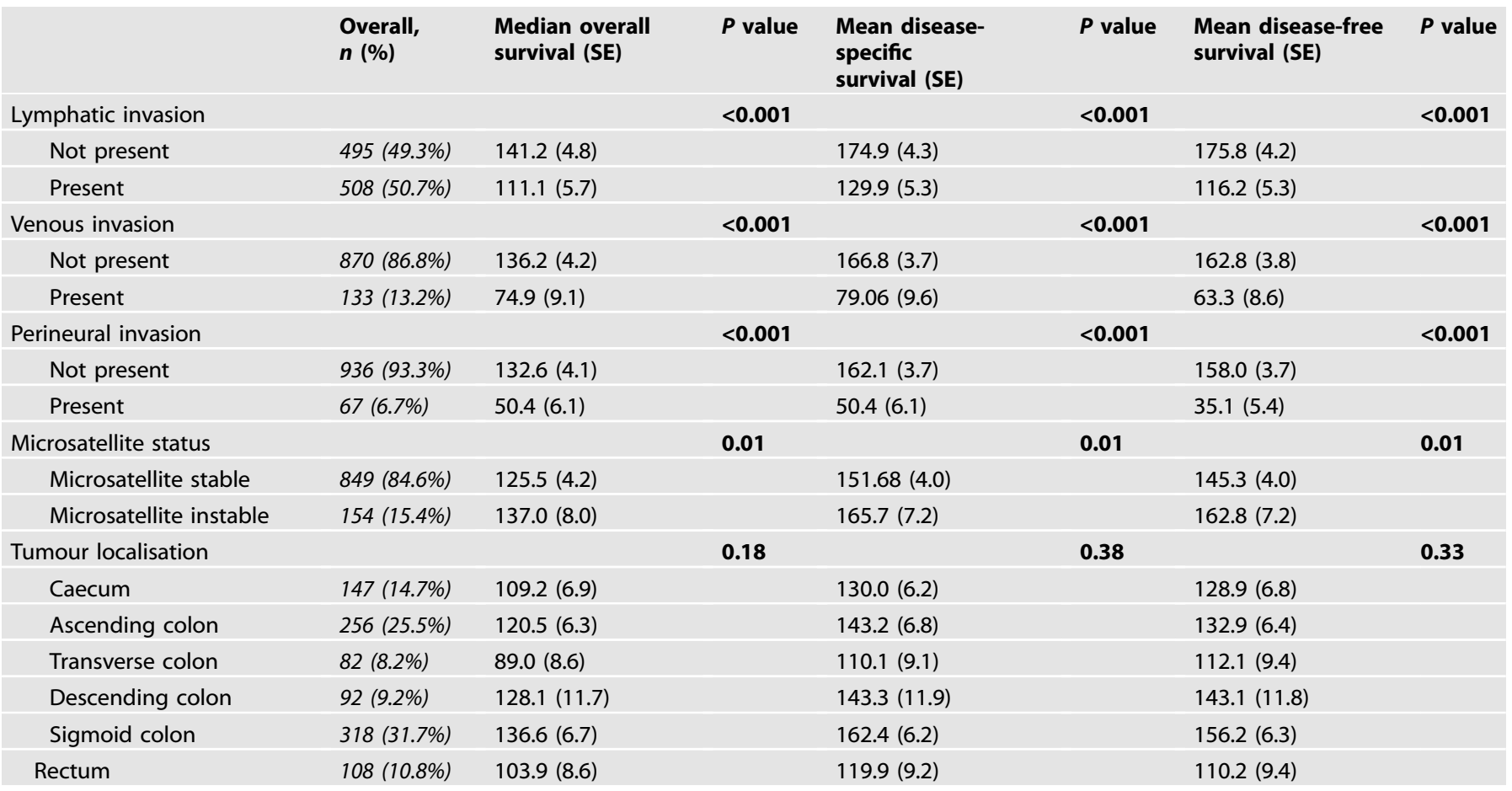

Bold values indicate statistical significance.

dichotomous CDX2 groups comparable to previous studies, which was guided using the Cutoff Finder [29]. Afterwards, CRCs were assigned into two groups regarding their CDX2 expression (CDX2 groups): CRCs that showed a CRC expression that was on the 10th percentile and below (range: $0-60 \%$ of tumour cells; $n=102,10 \%$ ) were categorised as CDX2-low/absent and CRCs with a CDX2 expression above the 10th percentile (61-100\% tumour cells; $n=$ 901, 90\%) were categorised as CDX2- high. CDX2-low/absent CRCs usually showed a reduced CDX2 staining intensity (CDX2-low/ absent: strong staining intensity: 0/102 (0\%), medium staining intensity: 24/102 (23\%), weak staining intensity: 66/102 (65\%), absent staining: 12/102 (12\%); CDX2-high: strong staining intensity: 842/901 (94\%), medium staining intensity: 59/901 (6\%), $P<0.001)$ and a significantly higher rate of a heterogeneous/ absent staining pattern when compared to CDX2-high tumours (CDX2-low/absent: absent: 12/102 (12\%), heterogeneous: 90/102 (88\%); CDX2-high: heterogeneous: $26 / 901$ (3\%), diffuse: $875 / 901$ (97\%), $P<0.001)$. A comparison of the results of the CDX2 grouping from 20 randomly selected full block slides showed a $100 \%$ concordance with the results from the TMA $(P<0.001)$. Furthermore, the interobserver variance between the two observers was assessed in 150 randomly selected cases, where an excellent correlation for the respective CDX2 groups was evident $(P<0.001$, Kappa-Cohens value: 0.84$)$.

\section{Distribution of morphologic parameters (CRC subtypes/ tumour budding/WHO grade) and microsatellite status}

CRC subtypes were present in the following numbers: 630 adenocarcinomas NOS (63\%), 122 micropapillary adenocarcinomas (12\%), 87 serrated adenocarcinomas (9\%), 83 mucinous (8\%) adenocarcinomas, 33 adenoma-like adenocarcinomas 3\%), 32 medullary carcinomas (3\%), 9 signet-ring cell carcinomas (1\%), 7 (1\%) mixed adenoneuroendocrine carcinomas (MANEC)/neuroendocrine carcinomas (NEC). A low tumour budding activity (Bd1) was observed in 545 CRCs (54\%), intermediate tumour budding $(\mathrm{Bd} 2)$ was detected in 261 tumours (26\%), a high tumour budding activity (Bd3) was observed in 197 cases (20\%). Six hundred and eighty-five CRCs were graded as low grade (68\%) and 318 as high grade (32\%) according to the WHO-grading system. Eighthundred and forty-nine CRCs were microsatellite stable (MSS; $849 / 1003,85 \%)$ and 154 were classified as microsatellite instable (MSI-High: 154/1003, 15\%, details Table 1).

Association of CDX2 groups with tumour stage, morphologic parameters (CRC subtypes/tumour budding/WHO grade) and microsatellite status

As depicted in detail in Fig. 1 and Table 2, CDX2-low/absent CRCs were significantly more frequent in higher $\mathrm{pT} / \mathrm{pN} / \mathrm{UICC}$-stages $(P<$ $0.001, P=0.039, P<0.001$, respectively) and right-sided tumours $(P<0.001)$. Compared to CDX2-high neoplasms, CDX2-low/absent $\mathrm{CRCs}$ were also more frequent in high-grade CRCs according to the WHO grade and tumours with intermediate $(\mathrm{Bd} 2)$ or high (Bd3) tumour budding activity as well as in MSI-H CRCs $(P<0.001$, respectively). However, the majority of cases with either increased tumour budding activity or high WHO grade fell into the CDX2high category.

The presence of CDX2-low/absent tumours in general was also significantly associated with the presence of certain histopathological CRC subtypes from both ends of the spectrum of biological aggressiveness $(P<0.001)$. For example, $>50 \%$ of the prognostically favourable (and frequently microsatellite-instable) medullary carcinomas as well as almost all of the biologically highly aggressive MANEC/NECs fell into the CDX2-low/absent subgroup.

\section{Prognostic relevance of CDX2 groups in the overall cohort, microsatellite subgroups, right- vs. left-sided tumours and UICC Stage II/III CRCs}

As illustrated in Fig. 2, Supplementary Fig. 1 and Table 1, compared to CDX2-high tumours, the CDX2-low/absent group showed a decreased OS (CDX2-high 131.2 months vs CDX2-low/ absent 100.3 months, $P=0.017$ ), DSS (CDX2-high 158.7 months vs CDX2-low/absent 121.9 months, $P=0.006)$ and DFS (CDX2-high: 
a
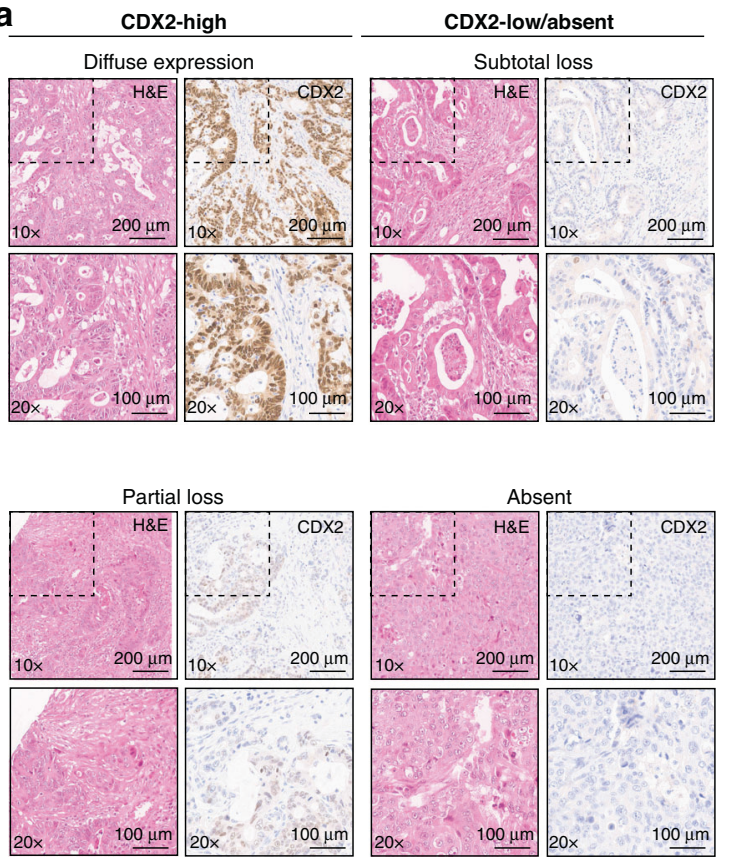

\section{f}

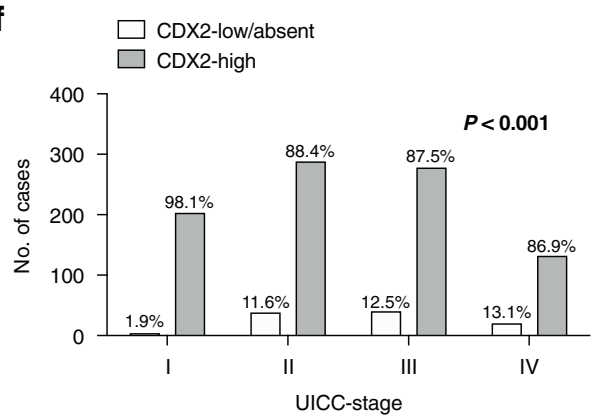

i

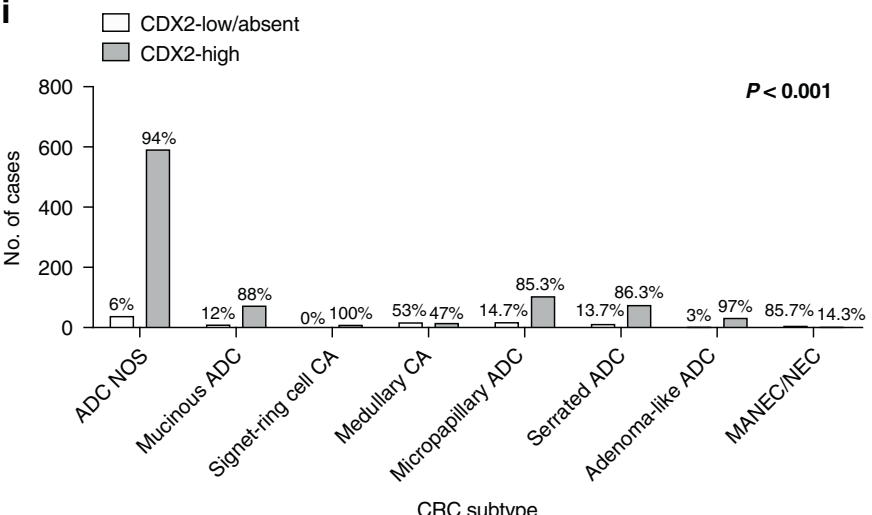

$\square$ CDX2-low/absent

CRC subtype j
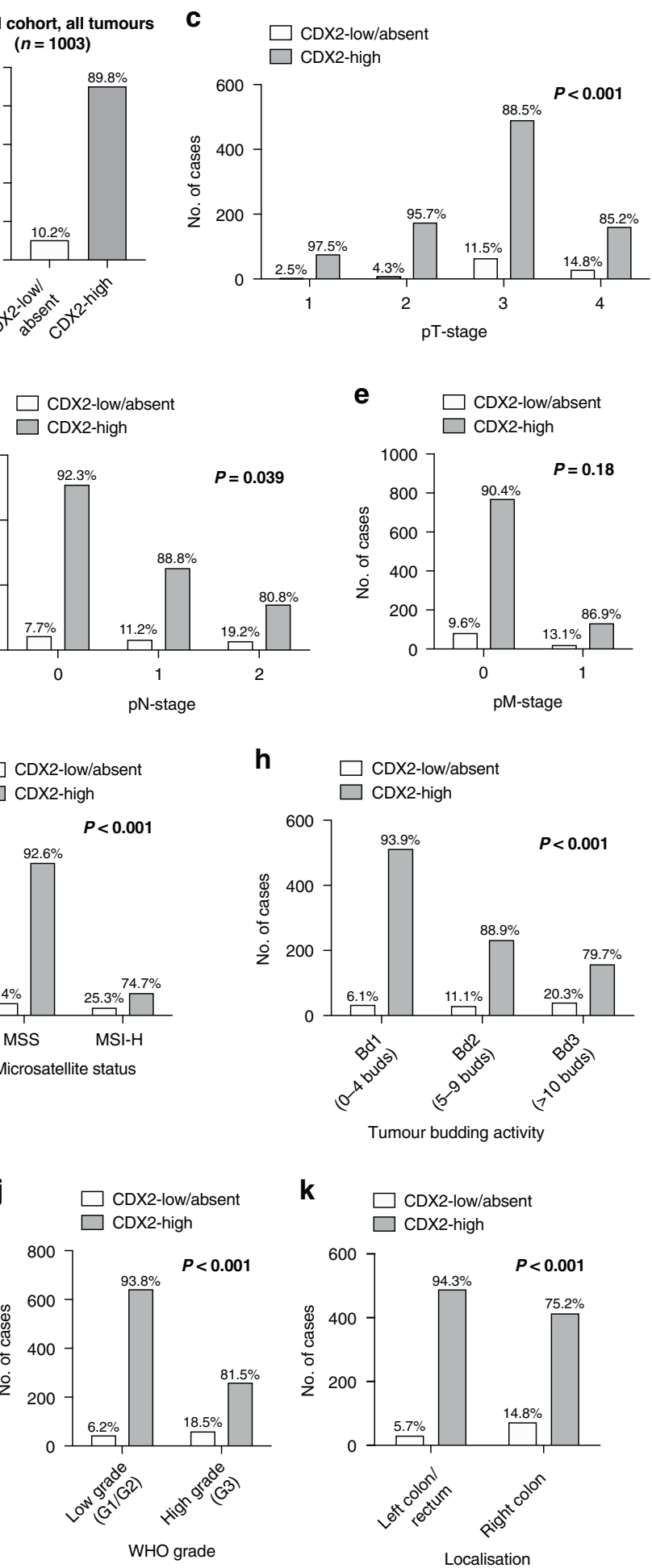

d
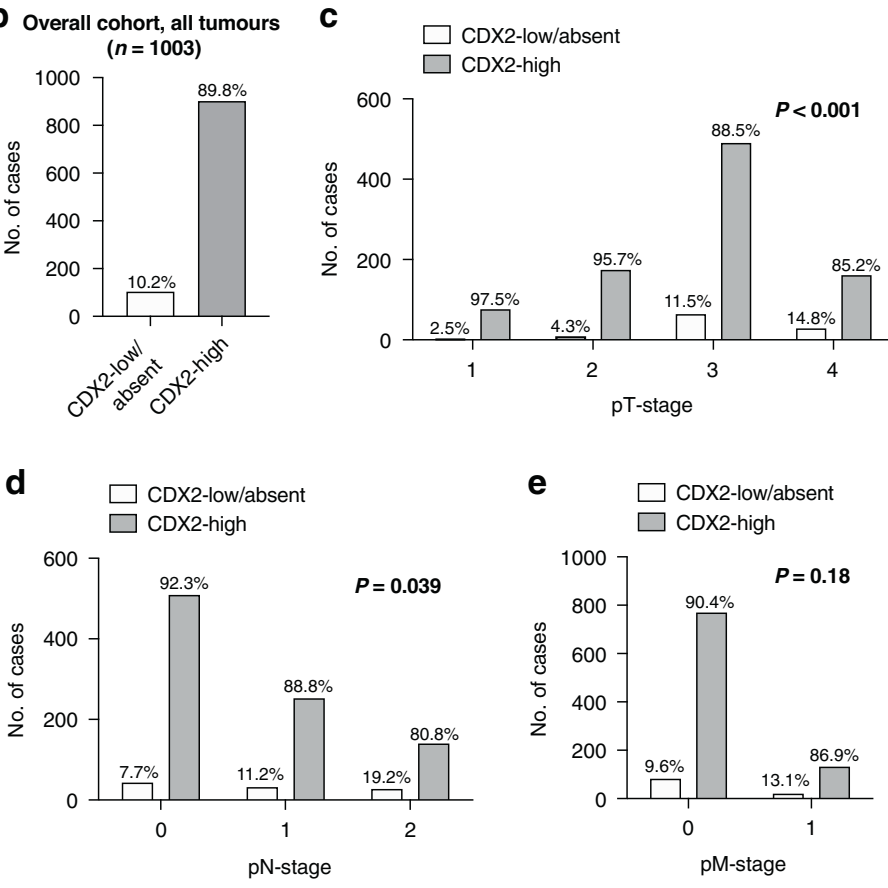

g

$\square$ CDX2-high

h

$\square$ CDX2-low/absent
$\square$ cDX2-high
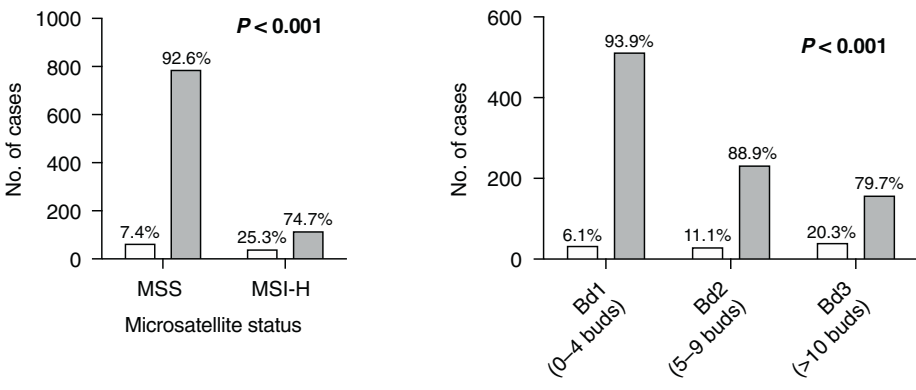

Tumour budding activity

k

k $\square$ cDX2-low/absent
$\square$ cDX2-high
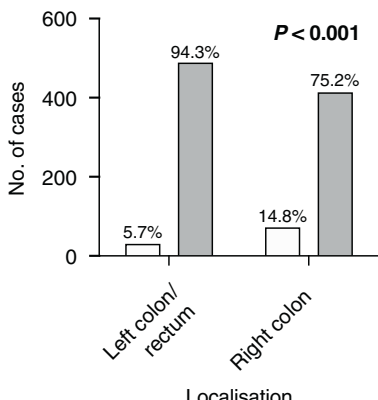

Fig. 1 Histopathology of CDX2-expression groups and distribution of CDX2 groups with HE-based morphological factors and clinicopathological parameters. Histopathology (a) and frequency of CDX2-expression groups (b) as well as the association of CDX2expression groups with pTNM status (c-e), UICC stage (f) and microsatellite status $(\mathbf{g})$ in the overall cohort. Further relationship of CDX2expression groups with tumour budding subgroups (h), CRC subtypes (i) and WHO grade (j) as well as tumour localisation (k) in the overall cohort. ADC adenocarcinoma, CA carcinoma. 
Table 2. Association of CDX2 expression with clinicopathological parameters as well as CRC subtypes, tumour budding and WHO grade in the overall cohort.

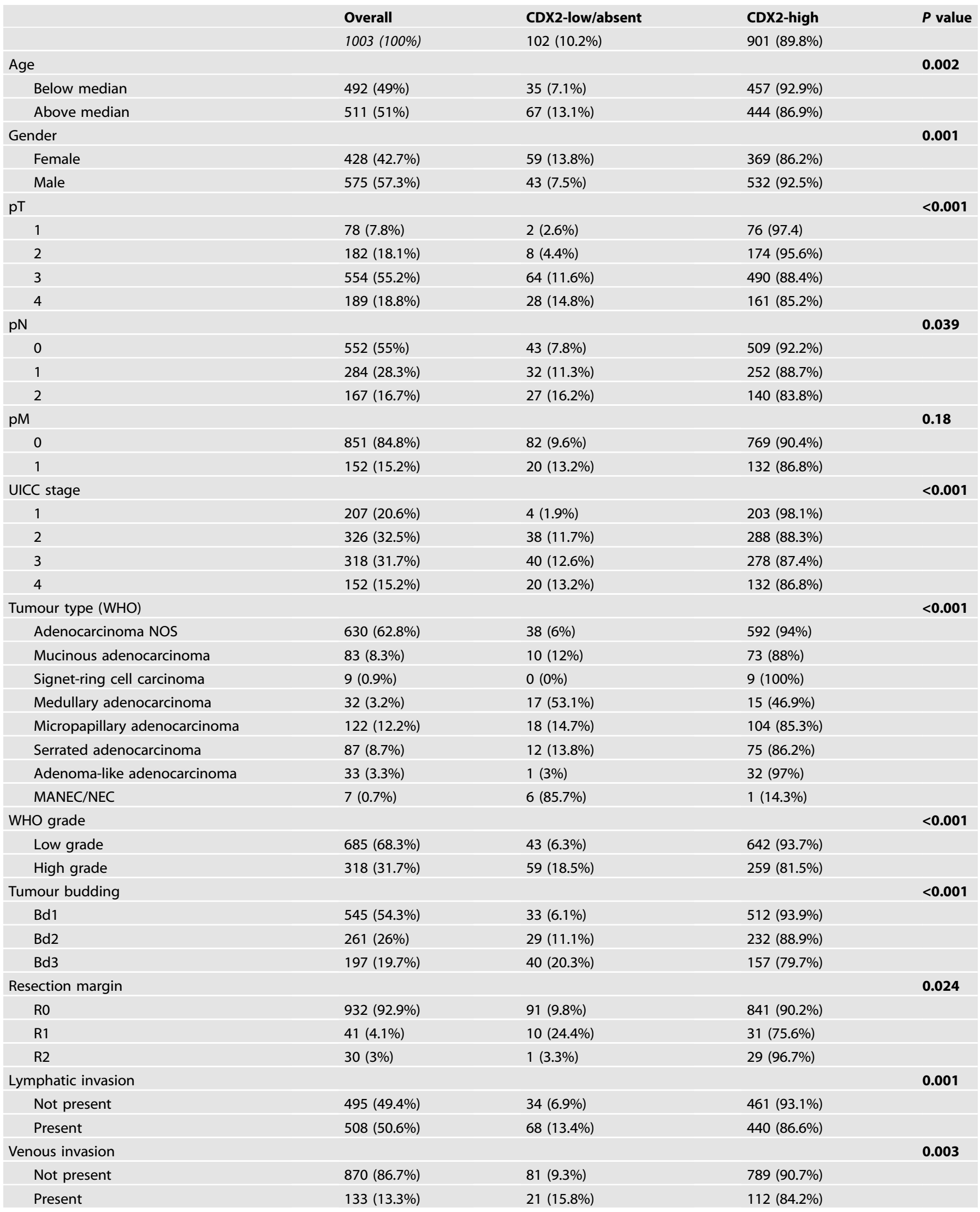


Table 2 continued

\begin{tabular}{|c|c|c|c|c|}
\hline & Overall & CDX2-low/absent & CDX2-high & $P$ value \\
\hline Perineural invasion & & & & 0.29 \\
\hline Not present & $936(93.3 \%)$ & $98(10.5 \%)$ & $838(89.5 \%)$ & \\
\hline Present & $67(6.7 \%)$ & $4(6 \%)$ & $63(94 \%)$ & \\
\hline Microsatellite status & & & & $<0.001$ \\
\hline Microsatellite stable & 849 (84.6\%) & $63(7.4 \%)$ & $786(92.6 \%)$ & \\
\hline Microsatellite instable & 154 (15.4\%) & 39 (25.3\%) & $115(74.7 \%)$ & \\
\hline Caecum & 147 (14.7\%) & $22(15 \%)$ & $125(85 \%)$ & \\
\hline Ascendening colon & $256(25.5 \%)$ & $43(16.8 \%)$ & $213(83.2 \%)$ & \\
\hline Transverse colon & $82(8.2 \%)$ & 7 (8.5\%) & 75 (91.5\%) & \\
\hline Descending colon & $92(9.2 \%)$ & $9(9.8 \%)$ & $83(90.2 \%)$ & \\
\hline Sigmoid colon & 318 (31.7\%) & $13(4.1 \%)$ & 305 (95.9\%) & \\
\hline
\end{tabular}

Bold values indicate statistical significance.

153.9 months vs CDX2-low/absent 114.9 months, $P=0.005)$ in the overall cohort of 1003 CRCs. As depicted in Table 3, the decreased survival of the CDX2-low/absent group was pronounced in the subgroup of MSS-CRCs ( $n=849$; OS: CDX2-high 127.4 months vs CDX2-low/absent 87.1 months, $P=0.012$; DSS: CDX2-high 154.6 months vs CDX2-low/absent 94.7 months, $P<0.001$; DFS: CDX2-high 148.2 months vs CDX2-low/absent 87.5 months, $P<$ 0.001 ), but was not significant in the subgroup of MSI-high CRCs $(n=154 ;$ OS/DSS/DFS, $P>0.05)$.

When analysed only in UICC Stage II/III CRCs $(n=644$, Fig. 2, Supplementary Fig. 2 and Table 4), CDX2-low/absent CRCs had a slightly lower DFS (CDX2-high 157.8 months vs CDX2-low/absent 148.1 months, $P=0.05$ ), but no significant differences were observed regarding OS/DSS ( $P>0.05$, respectively) between CDX2 groups. In microsatellite stable UICC Stage II/III CRCs, CDX2-low/absent tumours were associated with a significantly shorter DSS/DFS but not OS ( $n=525$; OS: $P>0.05$; DSS: CDX2-high 160.7 months vs CDX2-low/absent 108.8 months, $P=0.05$; DFS: CDX2-high 154.4 months vs CDX2-low/absent 96.8 months, $P=$ 0.009), while in microsatellite-instable UICC Stage II/III CRCs, CDX2 expression had no impact on any survival parameter (OS/DSS/DFS, $P>0.05$ ). When analysed in left- vs. right-sided CRCs, we observed a strong impact of CDX2 loss in univariate analyses of left-sided CRCs $(P<0.001$ for all comparisons, Supplementary Fig. 3$)$, which was also preserved in the subgroup of MSS left-sided CRCs $(P<$ 0.001 for all comparisons) and MSI-H left-sided CRCs (e.g: DFS, $P<$ 0.004). In right-sided CRCs, no prognostic effect of CDX2 was visible ( $P>0.05$ for all comparisons, data not shown).

When only adenocarcinomas NOS, the most common CRC subtype, were analysed, comparable survival results to the overall cohort were observed in all subgroups (e.g. DSS: $P=0.001$ in all adenocarcinomas NOS, data not shown). No significant survival differences were observed between CDX2-high vs CDX2-low/absent tumours within the different tumour budding $\mathrm{WHO}$ grade subgroups.

Prognostic impact of HE-based morphologic parameters (CRC subtypes/tumour budding/WHO grade) in the overall cohort, microsatellite subgroups, right- vs. left-sided tumours and in UICC Stage II/III CRCs

Compared to the different CDX2 expression groups, the central $\mathrm{HE}$-based morphologic parameters generally showed stronger survival discrimination than the CDX2-expression groups in the overall cohort and in Microsatellite subcohorts as well as UICC Stage 2/3 subgroups [8]. As depicted in detail in Table 1 and Supplementary Fig. 4, CRC subtypes strongly impacted on patient survival and showed a great variation regarding their OS/DSS/DFS ( $P<0.001$ for OS/DSS/DFS, respectively), with some subtypes like medullary carcinoma or adenoma-like adenocarcinoma showing a very indolent disease course and other specific variants like micropapillary adenocarcinoma or MANEC/NEC showing dismal survival characteristics. The different tumour budding categories (e.g. DFS: 190.6 months (Bd1) vs. 113.8 months (Bd2) vs. 48.5 $(\mathrm{Bd} 3))$ and the respective WHO-grades (e.g. DFS: 155.8 months (low grade) vs. 120.6 months (high grade)) were also strongly associated with patient survival on all survival comparisons $(P<$ 0.001 , respectively, for OS/DSS/DFS). When analysed separately in MSS- and MSI-H CRCs in the overall cohort, tumour budding $(P<$ 0.001 for all comparisons in MSS/MSI-H) and CRC subtypes (DSS: $P$ $<0.001$ in MSS; $P=0.007$ in MSI-H/DFS: $P<0.001$ in MSS; $P=0.01$ in MSI-H) retained their prognostic impact in both microsatellite subgroups, while comparable to $\mathrm{CDX} 2$, WHO grade only remained significant in MSS CRC $(P<0.001$ for all comparisons in MSS; $P>$ 0.05 for MSI-H, details Table 3 ).

When analysed in UICC Stage II/III only, similar effects on patient survival were noted. Tumour budding $(P<0.001$ for all comparisons in MSS/MSI-H) and CRC subtypes (DSS: $P<0.001$ in MSS; $P=$ 0.003 in MSI-H/DFS: $P<0.001$ in MSS; $P=0.009$ in MSI-H) retained their prognostic impact MSS and MSI-H UICC Stage II/III CRCs, while WHO grade only remained significant in MSS UICC Stage II/III CRCs (DSS: $P=0.001 / D F S: P<0.001$ in MSS; $P>0.05$ for MSI-H in all comparisons, Fig. 2, Supplementary Fig. 4 and Table 4). In left-/ right-sided CRCs as well as in the largest CRC subtype group (adenocarcinomas NOS), all parameters retained their strong prognostic impact (left-sided/right-sided CRCs/adenocarcinomas NOS only: $P<0.001$ for all comparisons; data not shown). Notably, when analysed separately within CDX2-high vs CDX2-low/absent tumours, tumour budding/CRC subtypes remained highly prognostic (e.g. DFS: $P<0.001$; data not shown) in all CDX2 groups, while WHO grade remained highly prognostic in CDX2-high (e.g. DFS: $P<0.001$; data not shown), but not in CDX2-low/absent tumours.

\section{Multivariate analyses}

In multivariate analyses (including age, gender, UICC stage, MSIstatus, WHO grade, tumour budding, CRC subtypes and CDX2 groups) CDX2 expression was not an independent prognostic factor in the overall cohort (DSS: $P=0.97,5$; Table 5; DFS: $P=0.75$, Supplementary Table 1; OS: $P=0.61$, Supplementary Table 2 ) and in the subcohorts of UICC Stage II/III CRCs (DFS: $P=0.58$; Supplementary Table 3; DSS: $P=0.72$, Supplementary Table 4; 

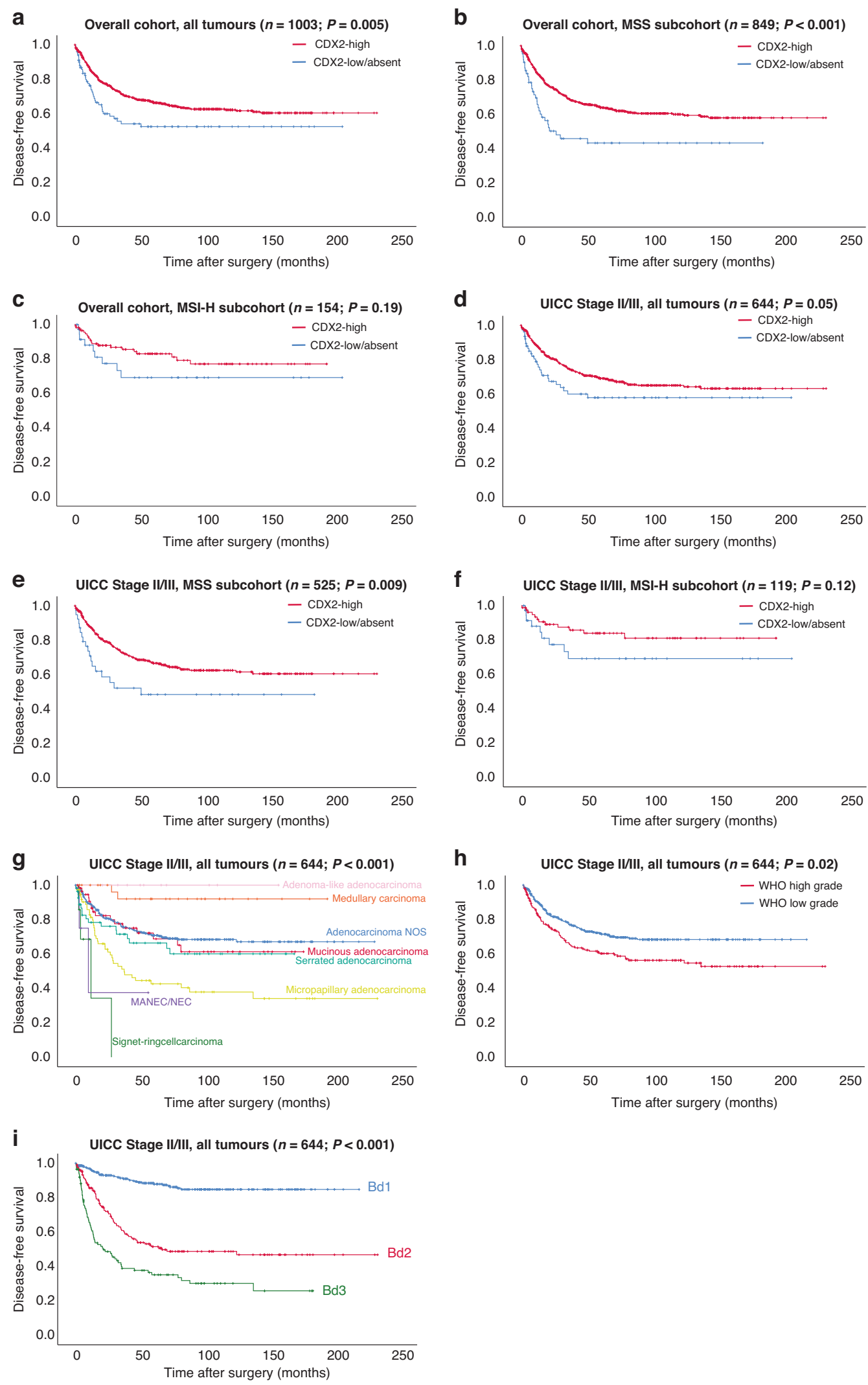

Fig. 2 Prognostic relevance of CDX2 and HE-based morphologic factors. Prognostic relevance of CDX2 expression groups on disease-free survival in the overall cohort including microsatellite subgroups $(\mathbf{a}-\mathbf{c})$, UICC Stage II/III tumours including microsatellite subgroups (d-f) and of the central HE-based morphologic factors CRC subtypes, WHO grade and tumour budding in all UICC Stage II/III tumours (g-i). 


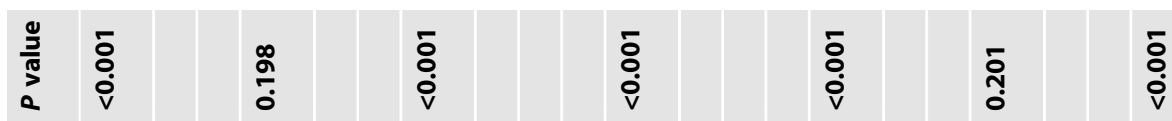

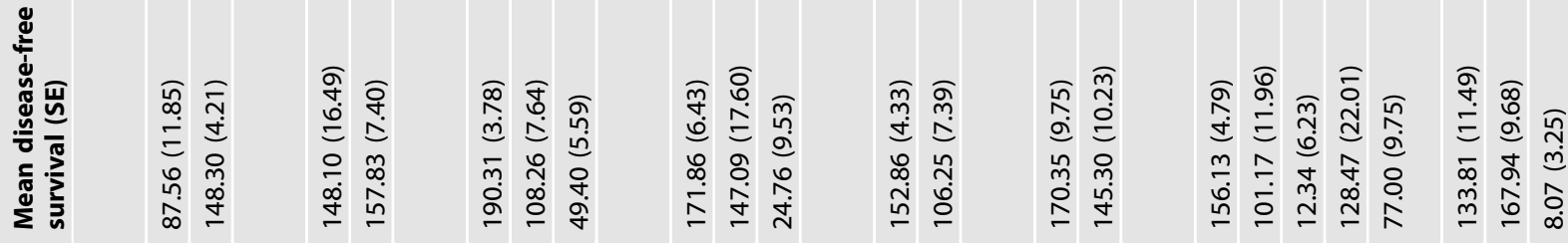

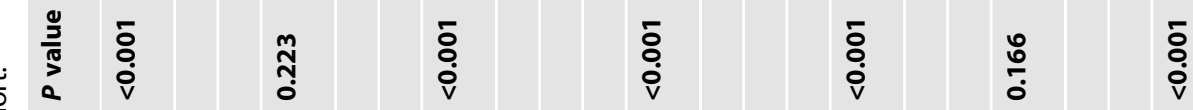

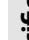

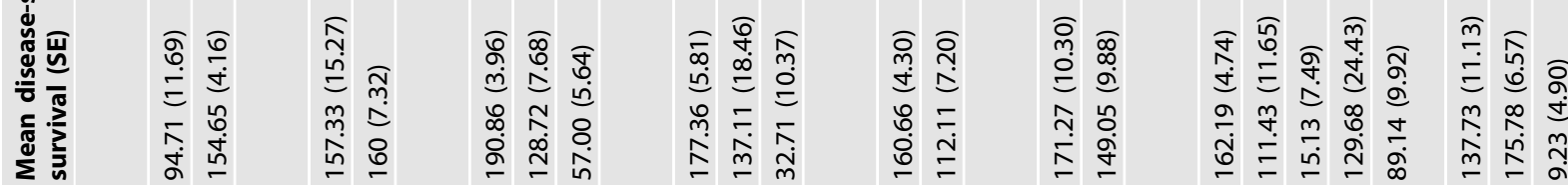

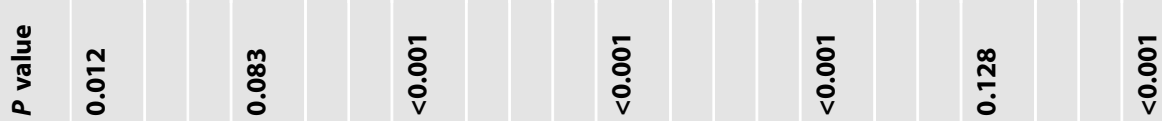

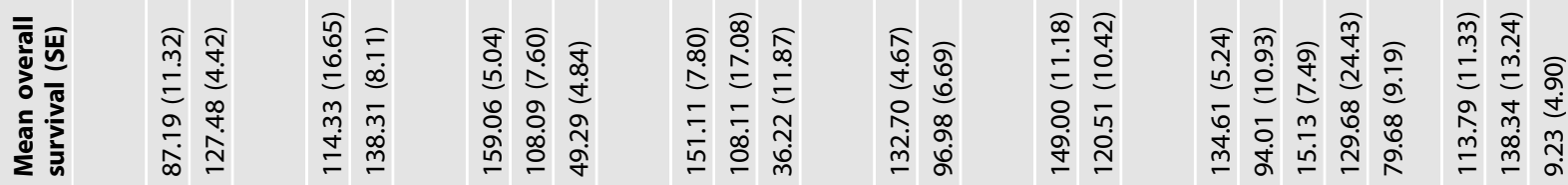
(1)

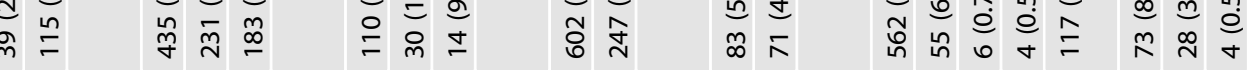

吾

$\ddot{\mho}$

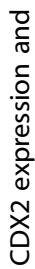

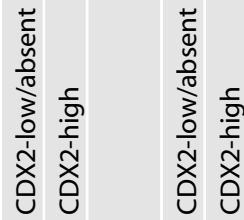

ธ்ָ

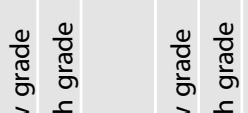

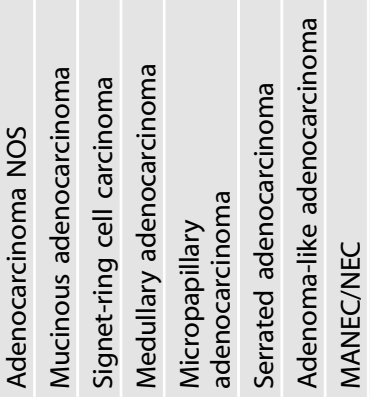

范

若

$\stackrel{0}{\underline{\sigma}}$

$\tilde{n}^{\tilde{n}}$

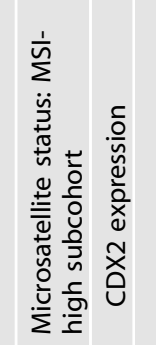

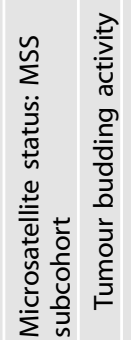

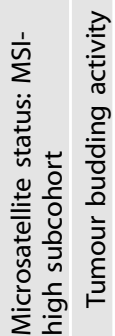
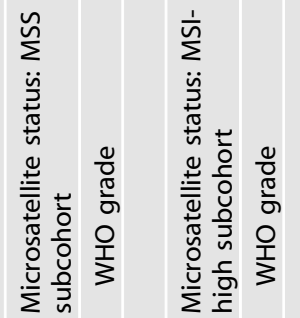

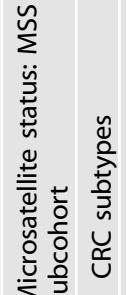


OS: $P=0.97$, Supplementary Table 5$)$ as well as in right-/left-sided CRCs ( $P>0.05$ for all comparisons, data not shown).

\section{DISCUSSION}

In this study, we evaluated the immunohistochemical expression of caudal-related homeobox transcription factor 2 (CDX2) in a large cohort of $>1000 \mathrm{CRCs}$ and correlated the results with staging parameters, microsatellite status and morphological parameters defined by the recent WHO classification (CRC subtypes, tumour budding, WHO grade). Finally, we analysed the prognostic relevance of CDX2 expression alone and compared it to the aforementioned histomorphologic parameters. Our study delivers three key messages: first, loss of CDX2 is of some prognostic relevance in CRC in univariate analyses, but its prognostic power is substantially inferior compared to the morphological factors defined by the WHO classification, especially when certain clinical subcohorts (e.g. UICC Stage II/III) are taken into account and vanishes when these parameters are incorporated into multivariate analyses. Second, CDX2 loss is massively enriched in MSI-H CRCs but fails to deliver prognostic information in this molecular subgroup. Third, loss of CDX2 is specifically enriched in certain CRC subtypes coming from both ends of the spectrum of biological aggressiveness, ranging from indolent variants such as medullary carcinomas to highly aggressive subtypes such as MANEC/NECs, arguing that both MSI-status, as well as the histomorphologic subtype of CRC, have to be considered before CDX2 might be used for clinical decision-making.

CDX2 is a highly conserved transcription factor that controls cell fate and differentiation in the intestinal epithelium and is diffusely expressed by non-neoplastic colonic epithelial cells [16]. CDX2 is also known to be strongly expressed by the majority of neoplasms of the colorectal system and is a marker that is frequently used in the daily practice of surgical pathology to verify or rule out an intestinal and especially colorectal origin of a carcinoma, for example in the setting of suspected CRC metastases in other organs or for histogenetic classification of cancers with unknown primary site [30]. For the diagnosis of primary CRC in resection specimen, CDX2 is not frequently used, because the vast majority of CRCs are accompanied by adenomatous precursor lesions that are diagnostic of primary CRC in combination with a morphologically compatible invasive carcinoma component. However, CDX2 gained increasing attention in the scientific community in recent years as some studies proposed a lost or diminished expression of CDX2 as an independent biomarker of a more aggressive disease course $[17,18,21,23,26]$, while other studies could not generally confirm these results [31-37]. Compared to CDX2, which is normally assessed via immunohistochemistry, the recent WHO classification from 2019 defines the different histomorphological subtypes of CRC, tumour budding activity and the traditional WHO-grading algorithm in its essential diagnostic criteria for CRC, which represent purely histomorphological parameters that are assessed through the evaluation of HE-stained slides. In a recent study, we were able to confirm the high prognostic and in part stage-independent (CRC subtypes/tumour budding) prognostic impact of these parameters in the very same CRC cohort of $>1000$ tumours [8].

Generally confirming previous data, we observed significantly shorter survival parameters for CRC patients whose tumours showed a CDX2 loss in univariate analyses in our first general screening of the overall cohort. However, the prognostic power of CDX2 in this overall screening approach was considerably lower than those of the different CRC subtypes, WHO grade and tumour budding, which all showed stronger discrimination of survival groups than CDX2 expression, generally picturing the aforementioned WHO parameters as superior prognostic biomarkers in unselected CRC cohorts. 


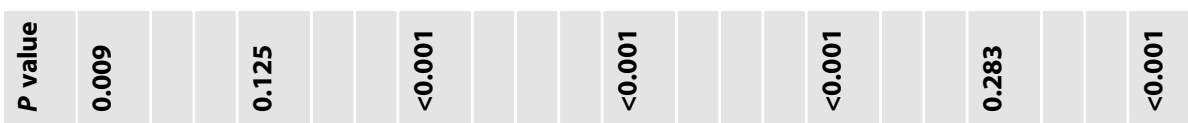

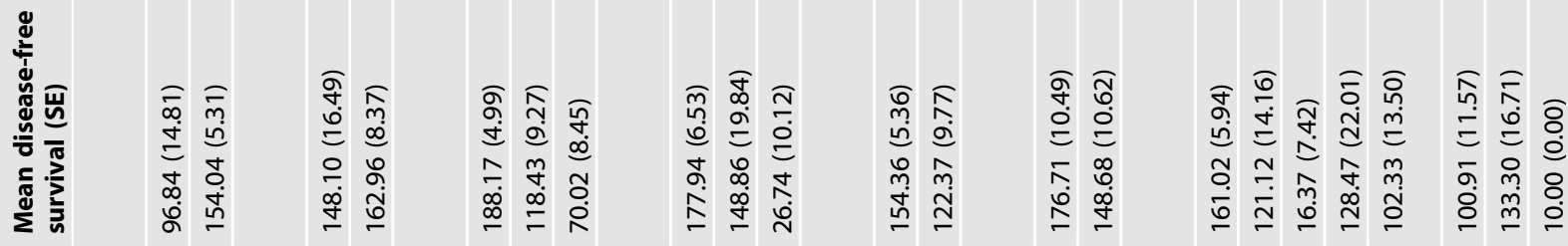

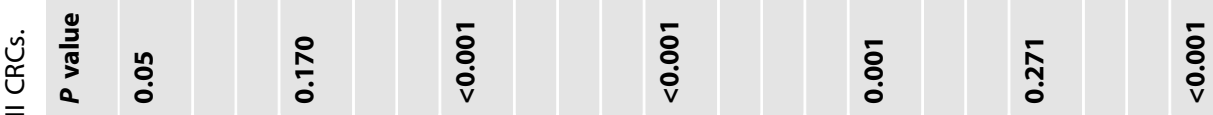

䯄

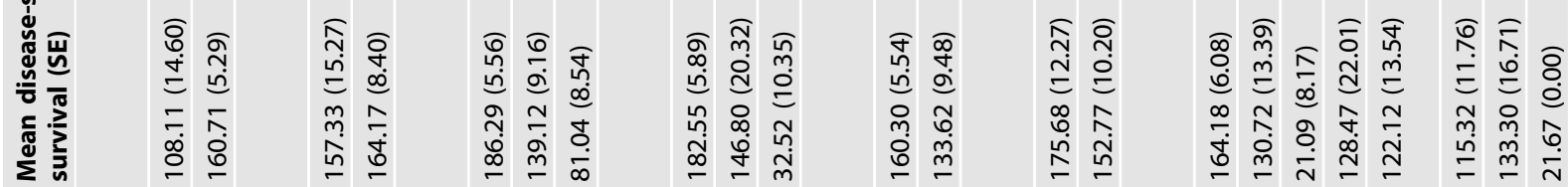







un

桀

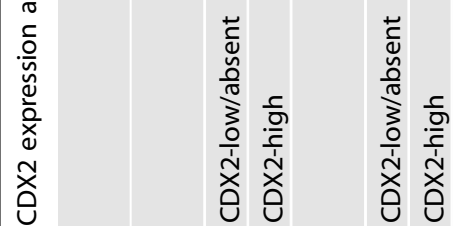

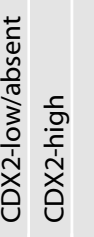

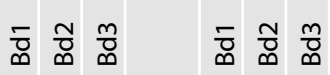

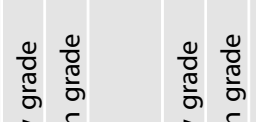

高旁

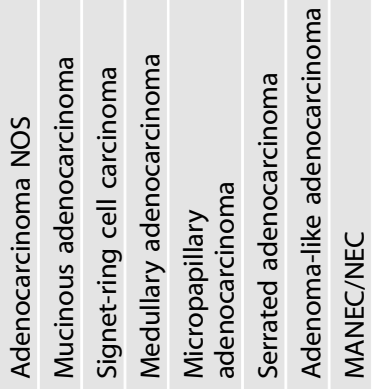

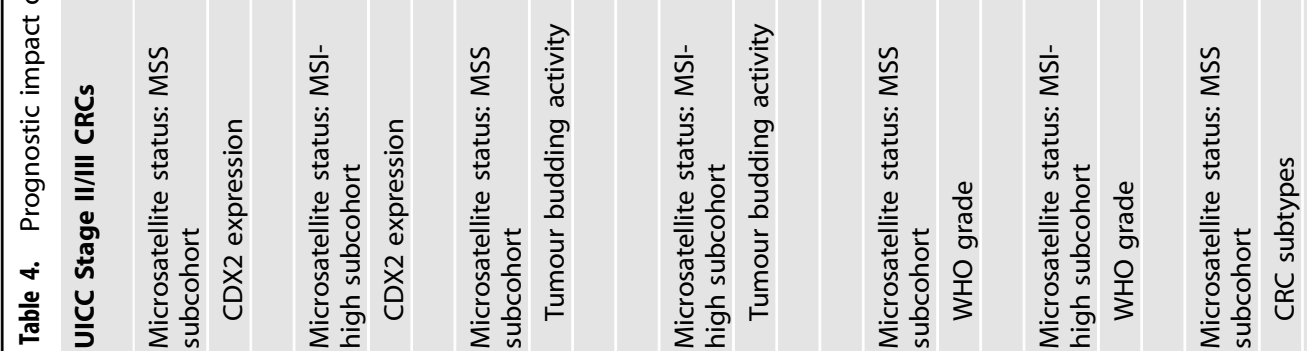


In the next step, we wanted to know if this general observation holds true when specific subcohorts of CRCs are investigated, which was the case when we analysed CRCs in UICC Stage II and III, where the prognostic power of CDX2 expression was also considerably lower compared to CRC subtypes, WHO grade and tumour budding. Although we can in principal confirm the results from previous studies such as the one from Dalerba et al. [17] that CDX2 is of some prognostic importance in univariate analyses in specific stage groups of CRC, our data for Stage II/III CRCs are comparable to the overall cohort, meaning that the purely morphological parameters allow for a significantly better assessment of different prognostic groups.

When we noticed the massive enrichment of CDX2 loss within MSI$\mathrm{H}$ tumours, we decided to look into the prognostic relevance of all parameters within microsatellite subgroups of the overall cohort and Stage II/III cancers and observed that CDX2 has a considerable prognostic impact in MSS tumours, but shows no prognostic relevance in MSI-H CRCs, where tumour budding and the different CRC subtypes (but not WHO grade) showed a high prognostic significance. These findings are in line with results from recent studies $[20,25,32,35]$ and argue that the prognostic relevance of CDX2 expression is tightly connected to the microsatellite status.

Another interesting observation of our study is the dependence of the prognostic impact of CDX2 to tumour localisation. The distinction of right- vs. left-sided CRCs by itself showed no general prognostic impact in our cohort, which is in line with findings from a large scale dataset analysed in a SEER study [38] and other studies [39, 40]. However, there are conflicting results in the literature regarding the general prognostic relevance of tumour localisation of CRC as some authors propose an improved survival for left-sided CRCs [41, 42]. In right-sided CRCs (caecum to splenic flexure), we observed no prognostic impact of CDX2 expression in contrast to a retained high prognostic relevance of CRC subtypes, tumour budding and WHO grade comparable to the data from the overall cohort. In left-sided CRCs (Descending colon to rectum), however, we observed acompared to the other subgroups-notably stronger prognostic impact of CDX2 loss on patient survival in univariate analyses, which was also present in left-sided microsatellite-instable CRCs, arguing that the evaluation of CDX2 expression has its highest relevance in leftsided colorectal carcinomas.

This general "sideness" of the prognostic impact of CDX2 expression may be related to the massive enrichment of CDX2-negative tumours in the medullary subtype of CRC, which is almost exclusively located in the right-sided colon and is associated with a comparatively benign clinical behaviour and thus probably narrows the potential worse prognostic effect of CDX2 loss in other CRC subtypes. Our findings argue towards the fact that not only the specific histologic subtype of CRC but also the tumour localisation and the microsatellite status have to be considered when the CDX2 status is assessed in order to determine patient prognosis.

In conclusion, our study confirms (but also significantly relativizes) the general prognostic relevance of CDX2 loss in colorectal cancer (in univariate analyses) and shows its association with tumour localisation, microsatellite status and certain CRC subtypes. Notably, CDX2 is not able to identify any additional subset of patients with a poor prognosis, that is not identified by either tumour budding, WHO grade or CRC subtypes. These central HE-based morphologic factors given by the WHO classification are generally prognostically superior compared to CDX2, with tumour budding being the strongest of the aforementioned parameters. These data suggest that these factors should be prioritised over CDX2 when histopathological parameters are used for clinical decision-making. The molecular mechanisms behind the enrichment of CDX2 loss in MSI-H tumours and rather benign (medullary $C R C$ ) or highly aggressive (MANEC/NEC) morphologic subtypes as well as in right-sided CRCs should be explored in further studies to potentially address potential therapeutic implications. 
Table 5. Multivariate disease-specific survival analysis in the overall cohort under inclusion of CDX2 expression, age, gender CRC subtype, tumour budding, WHO grade, UICC stage and microsatellite status.

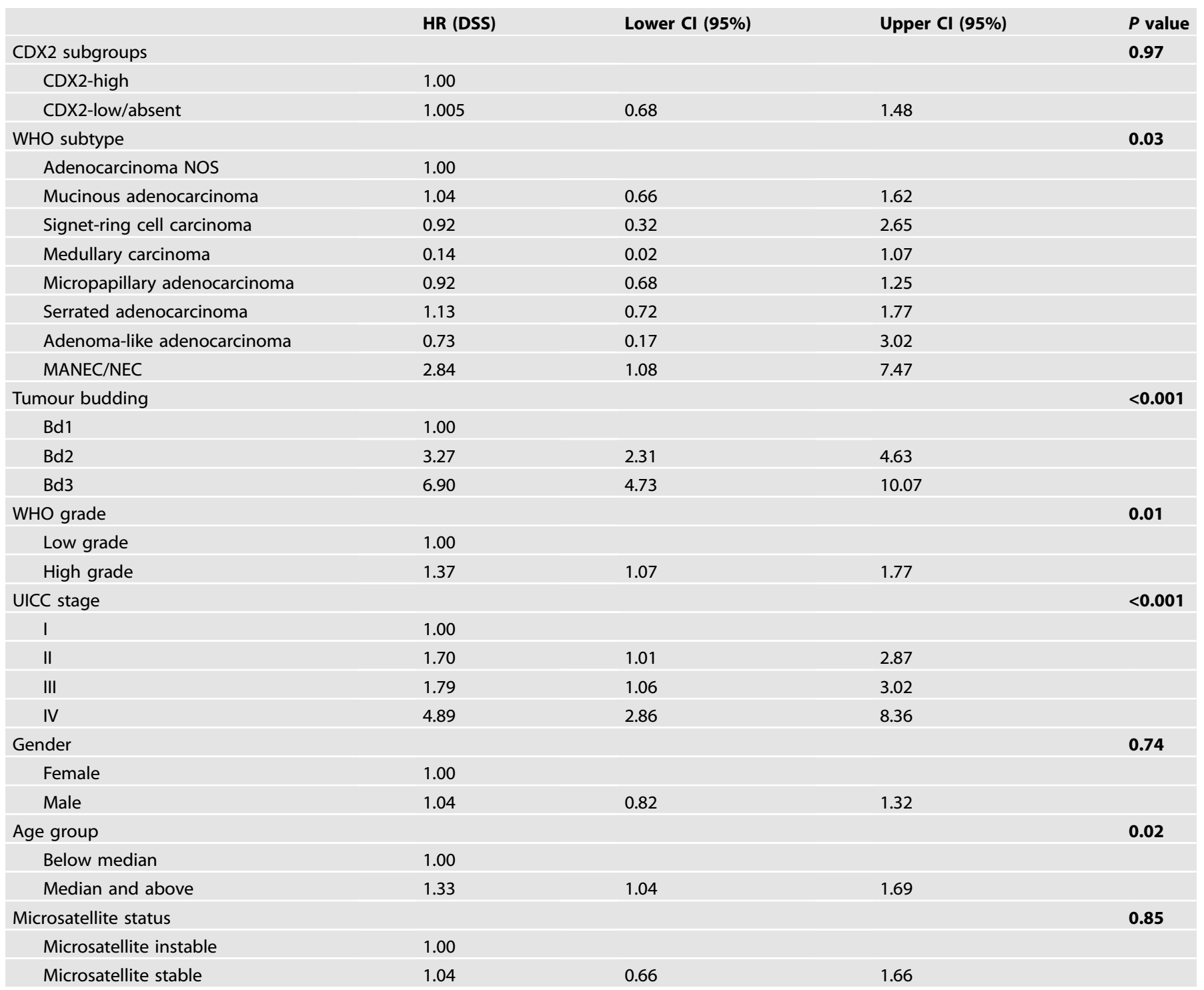

Bold values indicate statistical significance.

\section{DATA AVAILABILITY}

All data relevant for this study are given with the main paper including figures, tables and Supplemental files. The tissue investigated for this study is archived in the Institute of Pathology of the Technical University of Munich.

\section{REFERENCES}

1. Siegel RL, Miller KD, Goding Sauer A, Fedewa SA, Butterly LF, Anderson JC, et al. Colorectal cancer statistics, 2020. CA Cancer J Clin. 2020;70:145-64.

2. Sung H, Ferlay J, Siegel RL, Laversanne M, Soerjomataram I, Jemal A, et al. Global cancer statistics 2020: GLOBOCAN estimates of incidence and mortality worldwide for 36 cancers in 185 countries. CA Cancer J Clin. 2021;71:209-49.

3. Garcia-Solano J, Perez-Guillermo M, Conesa-Zamora P, Acosta-Ortega J, TrujilloSantos J, Cerezuela-Fuentes P, et al. Clinicopathologic study of 85 colorectal serrated adenocarcinomas: further insights into the full recognition of a new subset of colorectal carcinoma. Hum Pathol. 2010;41:1359-68.

4. Gonzalez RS, Cates JM, Washington MK, Beauchamp RD, Coffey RJ, Shi C. Adenoma-like adenocarcinoma: a subtype of colorectal carcinoma with good prognosis, deceptive appearance on biopsy and frequent KRAS mutation. Histopathology. 2016;68:183-90.
5. Haupt B, Ro JY, Schwartz MR, Shen SS. Colorectal adenocarcinoma with micropapillary pattern and its association with lymph node metastasis. Mod Pathol. 2007;20:729-33.

6. Hugen N, van de Velde CJ, de Wilt JH, Nagtegaal ID. Metastatic pattern in colorectal cancer is strongly influenced by histological subtype. Ann Oncol. 2014;25:651-7.

7. Hugen N, Verhoeven RH, Lemmens VE, van Aart CJ, Elferink MA, Radema SA, et al. Colorectal signet-ring cell carcinoma: benefit from adjuvant chemotherapy but a poor prognostic factor. Int J Cancer. 2015;136:333-9.

8. Jesinghaus $M$, Schmitt $M$, Lang $C$, Reiser $M$, Scheiter $A$, Konukiewitz $B$, et al. Morphology matters: a critical reappraisal of the clinical relevance of morphologic criteria from the 2019 WHO classification in a large colorectal cancer cohort comprising 1004 cases. Am J Surg Pathol. 2021;45:969-78.

9. Aramendi T, Fernandez-Acenero MJ, Villanueva MC. Carcinosarcoma of the colon: report of a rare tumor. Pathol Res Pr. 2003;199:345-8.

10. Knox RD, Luey N, Sioson L, Kedziora A, Clarkson A, Watson N, et al. Medullary colorectal carcinoma revisited: a clinical and pathological study of 102 cases. Ann Surg Oncol. 2015;22:2988-96.

11. Lee HJ, Eom DW, Kang GH, Han SH, Cheon GJ, Oh HS, et al. Colorectal micropapillary carcinomas are associated with poor prognosis and enriched in markers of stem cells. Mod Pathol. 2013;26:1123-31. 
12. Masoomi H, Ziogas A, Lin BS, Barleben A, Mills S, Stamos MJ, et al. Populationbased evaluation of adenosquamous carcinoma of the colon and rectum. Dis Colon Rectum. 2012;55:509-14.

13. Nagtegaal IDAM, Odze RD, Lam AK. Digestive system tumours: tumours of the colon and rectum, 5 edn. Lyon, France: International Agency for Research on Cancer (IARC); 2019.

14. Silberg DG, Sullivan J, Kang E, Swain GP, Moffett J, Sund NJ, et al. Cdx2 ectopic expression induces gastric intestinal metaplasia in transgenic mice. Gastroenterology. 2002;122:689-96.

15. Beck F, Chawengsaksophak K, Waring P, Playford RJ, Furness JB. Reprogramming of intestinal differentiation and intercalary regeneration in $\mathrm{Cd} \times 2$ mutant mice. Proc Natl Acad Sci USA. 1999;96:7318-23.

16. Moskaluk CA, Zhang H, Powell SM, Cerilli LA, Hampton GM, Frierson HF Jr. Cdx2 protein expression in normal and malignant human tissues: an immunohistochemical survey using tissue microarrays. Mod Pathol. 2003;16:913-9.

17. Dalerba P, Sahoo D, Paik S, Guo X, Yothers G, Song N, et al. CDX2 as a prognostic biomarker in stage II and stage III colon cancer. N. Engl J Med. 2016:374:211-22.

18. den Uil SH, de Wit M, Slebos RJC, Delis-van Diemen PM, Sanders J, Piersma SR, et al. Quantitative analysis of $\mathrm{CDX} 2$ protein expression improves its clinical utility as a prognostic biomarker in stage II and III colon cancer. Eur J Cancer. 2021;144:91-100.

19. Hansen TF, Kjaer-Frifeldt S, Eriksen AC, Lindebjerg J, Jensen LH, Sorensen FB, et al. Prognostic impact of CDX2 in stage II colon cancer: results from two nationwide cohorts. Br J Cancer. 2018;119:1367-73.

20. Hestetun KE, Aasebo K, Rosenlund NB, Muller Y, Dahl O, Myklebust MP. Mismatch repair phenotype determines the implications of tumor grade and CDX2 expression in stage II-III colon cancer. Mod Pathol. 2021;34:161-70.

21. Kim JH, Rhee YY, Bae JM, Cho NY, Kang GH. Loss of CDX2/CK20 expression is associated with poorly differentiated carcinoma, the CpG island methylator phenotype, and adverse prognosis in microsatellite-unstable colorectal cancer. Am J Surg Pathol. 2013;37:1532-41.

22. Lugli A, Tzankov A, Zlobec I, Terracciano LM. Differential diagnostic and functional role of the multi-marker phenotype CDX2/CK20/CK7 in colorectal cancer stratified by mismatch repair status. Mod Pathol. 2008;21:1403-12.

23. Ma C, Olevian D, Miller C, Herbst C, Jayachandran P, Kozak MM, et al. SATB2 and CDX2 are prognostic biomarkers in DNA mismatch repair protein deficient colon cancer. Mod Pathol. 2019;32:1217-31.

24. Pilati C, Taieb J, Balogoun R, Marisa L, de Reynies A, Laurent-Puig P. CDX2 prognostic value in stage II/III resected colon cancer is related to CMS classification. Ann Oncol. 2017;28:1032-5.

25. Slik K, Turkki R, Carpen O, Kurki S, Korkeila E, Sundstrom J, et al. CDX2 loss with microsatellite stable phenotype predicts poor clinical outcome in stage II colorectal carcinoma. Am J Surg Pathol. 2019;43:1473-82.

26. Xu W, Zhu Y, Shen W, Ding W, Wu T, Guo Y, et al. Combination of CDX2 expression and $T$ stage improves prognostic prediction of colorectal cancer. J Int Med Res. 2019;47:1829-42.

27. Lugli A, Kirsch R, Ajioka Y, Bosman F, Cathomas G, Dawson H, et al. Recommendations for reporting tumor budding in colorectal cancer based on the International Tumor Budding Consensus Conference (ITBCC) 2016. Mod Pathol. 2017;30:1299-311.

28. Brierley JD, Gospodarowicz MK, Wittekind C. TNM classification of malignant tumours. Hoboken, NJ, USA: John Wiley \& Sons; 2017.

29. Budczies J, Klauschen F, Sinn BV, Gyorffy B, Schmitt WD, Darb-Esfahani S, et al. Cutoff Finder: a comprehensive and straightforward Web application enabling rapid biomarker cutoff optimization. PLoS ONE. 2012;7:e51862.

30. Conner JR, Hornick JL. Metastatic carcinoma of unknown primary: diagnostic approach using immunohistochemistry. Adv Anat Pathol. 2015;22:149-67.

31. Altintas $S$, Bayrak M, Altintas $Y$. Prognostic value of $C D X 2$ and villin expression in advanced stage colorectal carcinoma. J Coll Physicians Surg Pak. 2019;29:1057-61.

32. Neumann J, Heinemann V, Engel J, Kirchner T, Stintzing S. The prognostic impact of CDX2 correlates with the underlying mismatch repair status and BRAF mutational status but not with distant metastasis in colorectal cancer. Virchows Arch. 2018;473:199-207.

33. Baba Y, Nosho K, Shima K, Freed E, Irahara N, Philips J, et al. Relationship of CDX2 loss with molecular features and prognosis in colorectal cancer. Clin Cancer Res. 2009;15:4665-73.

34. Olsen J, Eiholm S, Kirkeby LT, Espersen ML, Jess P, Gogenur I, et al. CDX2 downregulation is associated with poor differentiation and MMR deficiency in colon cancer. Exp Mol Pathol. 2016;100:59-66.

35. Ryan EJ, Creavin B, Khaw YL, Kelly ME, Mohan HM, Geraghty R, et al. Effects of CDX2 on prognosis and chemotherapy responsiveness in mismatch repairdeficient colorectal cancer. BJS Open. 2018;2:456-63.

36. Bremer FP, Czeczko NG, CollaCo LM, Rutz L, Gionedis G, Yamakawa CK. Are Cdx2 beta-catenin and wnt immunomarchers useful for evaluating the chance of disease progression or evolution to death in patients with colorectal cancer? Arq Bras Cir Dig. 2020;33:e1534.

37. Cecchini MJ, Walsh JC, Parfitt J, Chakrabarti S, Correa RJ, MacKenzie MJ, et al. CDX2 and Muc2 immunohistochemistry as prognostic markers in stage II colon cancer. Hum Pathol. 2019;90:70-79.

38. Weiss JM, Pfau PR, O'Connor ES, King J, LoConte N, Kennedy G, et al. Mortality by stage for right- versus left-sided colon cancer: analysis of surveillance, epidemiology, and end results-Medicare data. J Clin Oncol. 2011;29:4401-9.

39. Patel M, McSorley ST, Park JH, Roxburgh CSD, Edwards J, Horgan PG, et al. The relationship between right-sided tumour location, tumour microenvironment, systemic inflammation, adjuvant therapy and survival in patients undergoing surgery for colon and rectal cancer. Br J Cancer. 2018;118:705-12.

40. Powell AG, Wallace R, McKee RF, Anderson JH, Going JJ, Edwards J, et al. The relationship between tumour site, clinicopathological characteristics and cancerspecific survival in patients undergoing surgery for colorectal cancer. Colorectal Dis. 2012;14:1493-9.

41. Suttie SA, Shaikh I, Mullen R, Amin Al, Daniel T, Yalamarthi S. Outcome of rightand left-sided colonic and rectal cancer following surgical resection. Colorectal Dis. 2011;13:884-9.

42. Wray CM, Ziogas A, Hinojosa MW, Le $H$, Stamos MJ, Zell JA. Tumor subsite location within the colon is prognostic for survival after colon cancer diagnosis. Dis Colon Rectum. 2009:52:1359-66.

\section{ACKNOWLEDGEMENTS}

We thank the Comparative Experimental Pathology Unit of the Institute of Pathology and especially Marion Mielke and Olga Seelbach for excellent technical assistance. Furthermore, we thank Simone Köppel and Christina Schott from the Biobank of the Klinikum rechts der Isar (MTBio) for their excellent support.

\section{AUTHOR CONTRIBUTIONS}

$\mathrm{MJ}$ and BK designed this study. MJ and BK wrote the manuscript with assistance from SF, MT, WW. MJ, MS, WW and BK performed histopathological analysis. MJ, MiS, WW MT AMS and MB performed statistical analyses. MJ, MS, DW, KH, JP, JE, CL and NP collected clinicopathological data.

\section{FUNDING INFORMATION}

Funded by grants of the Deutsche Forschungsgemeinschaft (DFG, German Research Foundation): Project-ID 360372040 —SFB 1335 to WW and KS Project-ID 39535707SFB 1371. The authors have disclosed that they have no significant relationships with, or financial interest in, any commercial companies pertaining to this article. Open Access funding enabled and organized by Projekt DEAL.

\section{ETHICS APPROVAL AND CONSENT TO PARTICIPATE}

This study was performed in accordance with the Declaration of Helsinki and the local ethic committee of the Technical University of Munich approved this study (reference number: 252/16s).

\section{CONSENT TO PUBLISH}

Not applicable.

\section{COMPETING INTERESTS}

The authors declare no competing interests.

\section{ADDITIONAL INFORMATION}

Supplementary information The online version contains supplementary material available at https://doi.org/10.1038/s41416-021-01553-0.

Correspondence and requests for materials should be addressed to Moritz Jesinghaus.

Reprints and permission information is available at http://www.nature.com/ reprints

Publisher's note Springer Nature remains neutral with regard to jurisdictional claims in published maps and institutional affiliations. 
(c) (i) Open Access This article is licensed under a Creative Commons

Attribution 4.0 International License, which permits use, sharing, adaptation, distribution and reproduction in any medium or format, as long as you give appropriate credit to the original author(s) and the source, provide a link to the Creative Commons license, and indicate if changes were made. The images or other third party material in this article are included in the article's Creative Commons license, unless indicated otherwise in a credit line to the material. If material is not included in the article's Creative Commons license and your intended use is not permitted by statutory regulation or exceeds the permitted use, you will need to obtain permission directly from the copyright holder. To view a copy of this license, visit http://creativecommons. org/licenses/by/4.0/.

() The Author(s) 2021 